\title{
LATIICE GAUGE THEORIES
}

An Introduction 
This page is intentionally left blank 
World Scientific Lecture Notes in Physics - Vol. 43

\section{LATIICE GAUGE THEORIES An Introduction}

\section{Heinz J Rothe}

Institut für Theoretische Physik

Universitöt Heldelberg, Germany 


\title{
Published by
}

World Scientific Publishing Co. Pte. Ltd.

PO Box 128, Farrer Roed, Singapore 9128

USA office: Suite 1B, 1060 Main Street, River Edge, NJ 07661

UK office: 73 Lynton Mead, Tourridge, London N20 8DH

Library of Congress Cataloging-in-Publication Data is available.

Cover: 'CUBIC SPACE DIVISION (1952)' by M. C. Escher C 1952 M. C. Escher/Cordon An - Baam - Holland

\section{LATTICE GAUGE THEORIES - AN INTRODUCTION}

Copyright $\bigcirc 1992$ by World Scientific Publishing Co. Pte. Lid.

All rights reserved. This book, or parts thereof, may not be reproduced in any form or by any means, electronic or mechanical, including photocopying, recording or any information storage and retrieval system now known or to be invented, without written permission from the Publisher.

\author{
ISBN 981-02-0606-2 \\ 981-02-0607-0 (pbk)
}

Printed in Singapore by General Printing Services Pre. Lud. 


\section{To my wife Renate and to my children Stefan, Christine and Laura who taught me so many things about life}


This page is intentionally left blank 


\section{PREFACE}

This book is based on a one year course I held at the University of Heidelberg and on a series of lectures I gave at the "Autumn College on Techniques in Many-Body Problems" at Lahore, Pakistan, in November of 1987. These lectures have been published in the proceedings to this school by World Scientific (Rothe, 1989). I was later encouraged by the editors of World Scientific to expand on the material presented at the autumn College. This I have done in this book.

The purpose of my lectures at Lahore was to introduce lattice gauge theories to young physicists who may not have the opportunity to attend a course on this subject at their home universities. I had therefore kept the discussion as elementary as possible, including only enough technical details to enable the reader to follow the published literature on this subject. In this book I have expanded substantially on the material presented at Lahore, and have included a number of technical details which I felt would be very helpful to those readers who may want to carry out research in this branch of elementary particle physics. I did, however, arrange the material in such a way that those physicists who are mainly interested in getting a bird eyes view on the subject can safely skip the technical parts, without the danger of getting lost at a later stage. This concerns, in particular, the discussion in sections 4 and 5 of chapter 4 on lattice fermions, and the weak coupling expansion in lattice quantum chromodynamics (QCD), chapter 14. I have included this material for the readers convenience, since it is not discussed in such detail in the literature. I also decided to include a chapter on the path integral formalism, since the entire book is based on the path integral approach to quantization, and I do not assume that everybody is familiar with this formalism. Those readers that have never come in touch with the path integral formulation of quantum field theory may find this chapter a bit technical. However, the results we derive, of which we will make ample use in this book, are very simple, and are easily understood by everybody.

This book is mainly addressed to graduate students interested in particle physics. But it is also of interest to physicists actively engaged in research in the field of lattice gauge theories, and who may want to get a more general view on this subject. It assumes that the reader has a fair background in quantum field theory. A moderate knowledge of the continuum formulation of quantum chromodynamics would certainly be very helpful. Also physicists 
working in statistical mechanics may profit from reading this book, since the lattice formulation of field theories resembles closely that of complex statistical mechanical systems.

The book is divided into two parts. In the first part, comprising chapters 1 to $16, I$ discuss the zero temperature formulation of field theories on a spacetime lattice, and in particular QCD. They are the lattice analogues of the usual continuum field theories discussed in standard text books. The second part, consisting of chapters 17 to 20 , deals with finite temperature field theory. The emphasis will be on QCD, but I shall use ascalar field theory to introduce to the reader a number of new concepts which play an important role in finite temperature QCD.

Since the main goal of this book is to stimulate the readers interest in this fascinating branch of elementary particle physics, I have taken an optimistic stand-point, selecting some results of Monte Carlo calculations which illustrate the phenomena in a particularly dramatic way. I did not attempt to present a critical analysis of the results, and have left it to the reader to confer the original literature. Nor did I attempt to give a complete list of references, which the reader can find in the numerous proceedings to lattice conferences. More detailed discussions of most of the topics presented in this book can be found in the proceedings to various schools. An introduction to lattice gauge theories can also be found in the monograph by M. Creutz: Quarks, Gluons and Lattices, published by Cambridge University Press (1983).

Hopefully this book will stimulate some of the readers to carry out some research in the field of lattice gange theories. If so, I have achieved the purpose it has been written for.

I like to take this opportunity to thank a number of colleagues for their constructive criticisms and for having read several chapters of this book. In particular I am grateful to A. Actor, I. Bender, D. Gromes, F. Karsch, K. H. Mütter, I. O. Stamatescu and W. Wetzel. I am especially grateful to W. Wetzel for having checked a number of formulae, and for his extensive technical help in getting the manuscript into its final form. I also want to express my gratitude to Mrs. U. Einecke and Mrs. M. Steiert for having typed so patiently the manuscript in $T_{E} X$. Finally, I am particularily thankful to my family, whose continued support has made this book possible. In particular my children had to dispense of their father for many (!) hours. 


\section{CONTENTS}

Preface .................. . . vii

Chapter 1 INTRODUCTION ............... . 1

Chapter 2 THE PATH INTEGRAL APPROACH TO QUANTIZATION . . . . . . . . . . . . . . . 6

2.1 The Path Integral Method in Quantum Mechanics . . 7

2.2 Path Integral Representation of Bosonic Green Functions in Field Theory . . . . . . . . . . . . 13

2.3 The Transfer Matrix . . . . . . . . . . . . . . 20

2.4 Path Integral Representation of Fermionic Green Functions . . . . . . . . . . . . . . 22

2.5 Discretizing Space-Time. The Lattice as a Regulator of a Quantum Field Theory . . . . . . . . . . . 32

Chapter 3 THE FREE SCALAR FIELD ON THE LATTICE . . . . . . . . . . . . . . . . . . 35

Chapter 4 FERMIONS ON THE LATTICE . . . . . . . . . 42

4.1 The Doubling Problem . . . . . . . . . . . . . 42

4.2 Wilson Fermions . . . . . . . . . . . . . . . 47

4.3 Staggered Fermions . . . . . . . . . . . . . . 48

4.4 Technical Details of the Staggered Fermion Formulation . . . . . . . . . . . . . . . . . . 52

4.5 Staggered Fermions in Momentum Space . . . . 60

Chapter 5 ABELIAN GAUGE FIELDS ON THE LATTICE AND COMPACT QED . . . . . . . . 65

5.1 Preliminaries . . . . . . . . . . . . 65

5.2 Lattice Formulation of QED . . . . . . . . . . 68 
Chapter 6 NON-ABELIAN GAUGE FIELDS ON THE

LATTICE. COMPACT QCD . . . . . . . . . 75

Chapter 7 THE WILSON LOOP AND THE STATIC QUARK-ANTIQUARK POTENTIAL . . . . . . 83

7.1 A Look at Non-Relativistic Quantum Mechanics . 84

7.2 The Wilson Loop and the Static $q \bar{q}$-Potential in QED . . . . . . . . . . . . . . . . . . . . . . . 85

7.3 The Wilson Loop in QCD . . . . . . . . . . . 93

Chapter 8 THE $Q \bar{Q}$ POTENTIAL IN SOME SIMPLE MODELS . . . . . . . . . . . . . . . . 97

8.1 The Potential in Quenched QED _ . . . . . . . 97

8.2 The Potential in Quenched Compact QED $_{2}$. . 102

Chapter 9 THE CONTINUUM LIMIT OF LATTICE QCD . 107

9.1 Critical Behaviour of Lattice QCD and the Continuum Limit . . . . . . . . . . . . . . . 107

9.2 Dependence of the Coupling Constant on the Lattice Spacing and the Renormalization-Group $\beta$-Function . . . . . . . . . . . . 110

Chapter 10 THE STRONG COUPLING EXPANSION . . . . 118

10.1 The $q \bar{q}$-Potential to Leading Order in Strong Coupling . . . . . . . . . . . . . . . . . . . 118

10.2 Beyond the Leading Approximation . . . . . . . 121

10.3 The Lattice Hamiltonian in the Strong Coupling Limit and the String Picture of Confinement . . . 125

Chapter 11 THE HOPPING PARAMETER EXPANSION . . 137

11.1 Path Integral Representation of Correlation Functions in terms of Bosonic Variables . . . . . . . . . . 138

11.2 Hopping Parameter Expansion of the Fermion Propagator in an External Field . . . . . . . . . 141

11.3 Beyond the Quenched Approximation. HPE Expansion of the Effective Action . . . . . . 146

11.4 The HPE and the Pauli Exclusion Principle . . . . 151 
Chapter 12 WEAK COUPLING EXPANSION (I).

THE $\Phi^{3}$-THEORY . . . . . . . . . . . . . . . . 159

12.1 Introduction . . . . . . . . . . . . . . . . . 159

12.2 Weak Coupling Expansion of Correlation Functions in the $\phi^{3}$-Theory . . . . . . . . . . . . . . . 162

12.3 The Power Counting Theorem of Reisz . . . . . . 168

Chapter 13 WEAK COUPLING EXPANSION (II).

LATTICE QED

13.1 The Gauge Fixed Lattice Action . . . . . . . . . 176

13.2 Lattice Feynman Rules . . . . . . . . . . . . . 183

Chapter 14 WEAK COUPLING EXPANSION (III).

LATTICE QCD . . . . . . . . . . . . . . . . . . 190

14.1 The Link Integration Measure . . . . . . . . . . 191

14.2 Gauge Fixing and the Faddeev-Popov

Determinant . . . . . . . . . . . . . . . 195

14.3 The Gauge Field Action . . . . . . . . . . . . 200

14.4 Propagators and Vertices . . . . . . . . . . 205

14.5 Relation between $\Lambda_{L}$ and the $\Lambda$-Parameter of

Continuum QCD . . . . . . . . . 215

Appendix A

Appendix B . . . . . . . . . . . . . . . . . . . . 222

Chapter 15 MONTE CARLO METHODS . . . . . . . . 225

15.1 Introduction . . . . . . . . . . . . . . . . . 225

15.2 Construction Principles for Algorithms.

Markov Chains . . . . . . . . . . . . . . 227

15.3 The Metropolis Method . . . . . . . . . . . . 232

15.4 The Langevin Algorithm . . . . . . . . . . . . 234

15.5 The Molecular Dynamics Method . . . . . . 236

15.6 The Hybrid Algorithm . . . . . . . . . . . . . 242

15.7 The Hybrid Monte Carlo Algorithm . . . . . . . 245

15.8 The Pseudofermion Method . . . . . . . . . . . 248

15.9 Application of the Hybrid Monte Carlo Algorithm

to Systems with Fermions . . . . . . . . . . . 254 
xii Contents

Chapter 16 SOME RESULTS OF MONTE CARLO

CALCULATIONS

16.1 The String Tension and the $q \bar{q}$-Potential in the SU(3) Gauge Theory . . . . . . . . . . . . . 258

16.2 The $q \bar{q}$-Potential in Full QCD . . . . . . . . . . 265

16.3 Chiral Symmetry Breaking . . . . . . . . . . . 267

16.4 Glueballs . . . . . . . . . . . . . . . . . . 271

16.5 Hadron Mass Spectrum . . . . . . . . . . . . . 277

Chapter 17 INTRODUCTION TO FINITE TEMPERATURE FIELD THEORY . . . . . . . . . . . . . . . . 288

17.1 Introduction . . . . . . . . . . . . . . . 288

17.2 The Partition Function of the $\lambda \phi^{4}$ - Theory . . . . 290

17.3 Finite Temperature Feynman Rules . . . . . . . 293

17.4 Temperature Effects in the Two-Point Correlation Function . . . . . . . . . . . . . . . . . 303

17.5 Perturbative Expansion of the Thermodynamical Potential . . . . . . . . . . . . . . . 307

17.6 A Comment . . . . . . . . . . . 315

Chapter 18 LATTICE FORMULATION OF QCD AT FINITE TEMPERATURE . . . . . . . . . . . . 317

18.1 The Partition Function of QCD . . . . . . . . 317

18.2 Thermodynamical Observables on the Lattice . . . 322

18.3 The Wilson Line or Polyakov Loop . . . . . . . 326

18.4 Spontaneous Breakdown of the Center Symmetry and the Deconfinement Phase Transition . . . . 330

18.5 How to Determine the Transition Temperature . . 332

Chapter 19 MONTE CARLO STUDY OF THE

DECONFINEMENT AND CHIRAL PHASE TRANSITION 334

19.1 The Deconfinement Phase Transition in the Pure SU(3) Gauge Theory . . . . . . . . . . . 335

19.2 The Chiral Phase Transition . . . . . . . . . . 342 
Chapter 20 THE HIGH TEMPERATURE PHASE OF QCD . 347 20.1 Thermodynamical Potential in the Ideal Gas Approximation . . . . . . . . . . . . . . . . 347

20.2 Beyond the Ideal Gas Approximation. The Infrared Problem . . . . . . . . . . . . . . 349

20.3 Monte Carlo Study of the High Temperature Phase of QCD . . . . . . . . . . . . . . . . . . . 352

$20.4 J / \psi$ Suppression by a Plasma . . . . . . . . . . 359

CONCLUSION ................... . . 366

References .................. . . 369

Index . . . . . . . . . . . . . . . . 379 